\title{
MARIMBONDOS (HYMENOPTERA, VESPIDAE) COMO FONTE DE ALIMENTAÇÃO HUMANA NO BRASIL: UMA REVISÃO DE LITERATURA
}

\author{
WASPS (HYMENOPTERA, VESPIDAE) AS HUMAN FOOD IN BRAZIL: \\ A LITERATURE REVIEW
}

Ederson Tadeu BUENO, Brás Almendra Praça de CARVALHO, Marcos Magalhães de SOUZA*

Instituto Federal de Educação, Ciência e Tecnologia do Sul de Minas Gerais, Campus Inconfidentes,

Praça Tiradentes, 416, Centro, CEP 37576-000, Inconfidentes - MG *Autor correspondente: marcos.souza@ifsuldeminas.edu.br

\section{RESUMO}

Submitted: 08/06/2020; Accepted: 17/07/2020

Historicamente, insetos fazem parte da alimentação humana, prática também registrada no Brasil, especialmente em comunidades indígenas e tradicionais. Essa entomofagia inclui as vespas sociais, também conhecidas no Brasil como cabas ou marimbondos (Hymenoptera, Vespidae), e com ampla distribuição de espécies em todo o país. Partindo de uma revisão da literatura, o presente estudo lista as espécies de vespídeos sociais usadas como fonte de alimentação no Brasil. Realizou-se consulta bibliográfica utilizando diferentes plataformas de busca, entre agosto de 2019 a junho de 2020 . No Brasil há registro de seis espécies de vespas sociais consumidas por comunidades indígenas, sobretudo na região amazônica, e há ocorrência de outras 34 que são consumidas em outros países. O consumo desses animais na alimentação humana pode ser viável, sendo necessários estudos para criação e uso sustentável desse recurso natural.

Palavras-chave: Polistinae, entomofagia, Cabas

\begin{abstract}
Historically, insects are part of human diet a practice also registered in Brazil, especially among indigenous and traditional communities. This entomophagy includes social wasps, popularly known as cabas or marimbondos in Brazil, widely distributed in the country. Based on a literature review, the present study lists the species of social wasps used as a food source in Brazil. Bibliographic consultation was carried out from August 2019 to June 2020 by using different search platforms. In Brazil, there are records of six species of social wasps consumed by indigenous communities, especially in the Amazon region, and there are other 34 that are consumed in other countries. The consumption of these animals may be feasible, but requires further studies in terms of rearing and sustainable use of these natural resources.
\end{abstract}

Keywords: Polistinae, entomophagy, wasps

\section{INTRODUÇÃO}

A entomofagia consiste no uso de insetos na alimentação, hábito presente na cultura de mais de 100 países (MALAISSE, 1997; BANJO et al., 2004; RAMOS-ELORDUY, 2004; VAN HUIS, 2020). Sabe-se que já no Plio-Pleistoceno, os cupins faziam parte da dieta do hominídeo Australopithecus robustus (COSTA NETO, 2002). Diversas culturas africanas possuem o hábito de comer insetos, como exemplo o uso de algumas espécies de formigas misturadas em saladas, no intuito de obter um sabor característico, resultado do ácido fórmico produzido por esses insetos (NONAKA, 1996). Em países como México e Japão, a entomofagia é um costume cultural e as pessoas fazem uso de muitas espécies de insetos, dentre as quais as larvas de vespas (COSTA NETO, 2003).

No Brasil, a cultura de comer insetos tem origem indígena (COSTA NETO, 2000), mas esse hábito não se restringe aos índios. O consumo de formigas conhecidas por içás, saúvas ou tanajuras (fêmeas aladas de Atta spp.), é relatado em muitos Estados, como o Amazonas, São Paulo, Minas Gerais, Bahia e Ceará, onde inclusive há um festival em que celebra essa tradição (COSTA NETO, 2003). Além do consumo de formigas, há no território brasileiro o consumo de outros insetos, que na sua fase larval são utilizados como fonte de alimento, como besouros (Coleoptera) das espécies Pachymerus cardo (Fåhraeus, 1839), Caryobruchus sp., Rhynchophorus palmarum (Linnaeus, 1758), Rhina 
barbirostris (Fabricius, 1775) e Pachymerus nucleorum (Fabricius 1792); borboletas (Lepidoptera) das espécies Brassolis sophorae (Linnaeus, 1758) e Myelobia smerintha (Hübner, 1821); abelhas (Hymneoptera) das espécies Frieseomelitta silvestrii (Friese, 1902), Geotrigona mombuca (Smith, 1863), Melipona asilvai Moura, 1971 (COSTA NETO, 2000; COSTA NETO e RAMOS-ELORDUY, 2006; JONGEMA, 2017).

Os insetos, em sua grande maioria, contêm altas quantidades de proteínas e de lipídeos, são ricos em sódio, potássio, zinco, fósforo, manganês, magnésio, ferro, cobre e cálcio, e muitas espécies são ricas em vitaminas do grupo B, como tiamina (B1), riboflavina (B2) e niacina (B6). Além disso, os lipídios formadores das gorduras são, em sua grande maioria, insaturados ou poli-insaturados, portanto, necessários e benéficos ao organismo, podendo ser substitutos da carne bovina e de frango (COSTA NETO, 2003, 2004; SIRIMUNGKARARAT et al., 2010; RAMOS-ELORDUY et al., 2012). Além disso, esses invertebrados são mais eficientes na conversão de sua alimentação em relação a sua biomassa. Segundo a Organização das Nações Unidas para a Alimentação e a Agricultura (FAO), os insetos possuem uma relação de $2 \mathrm{~kg}$ de alimento para $1 \mathrm{~kg}$ de biomassa, enquanto bovinos possuem uma relação de $8 \mathrm{~kg}$ de alimento para $1 \mathrm{~kg}$ de massa corporal (VAN HUIS, 2013).

Do ponto de vista da sustentabilidade, os insetos são considerados uma fonte natural renovável e sua criação causa um impacto ambiental mínimo, em comparação com os causados pela pecuária e avicultura (COSTA NETO, 2003). Sendo assim, a entomofagia apresenta-se como uma boa aliada na conservação do meio ambiente e pode ajudar a diminuir o desmatamento (ROMERO, 2012), sendo que grande parte do solo da Terra vem sendo degradado com práticas de pecuária e agricultura insustentáveis (ROMERO, op. cit.).

A população humana está crescendo cerca de $2 \%$ por ano (RICKLEFS, 1996) e estima-se que em 2050 chegue ao número de nove bilhões; assim, os recursos ficarão ainda mais escassos para abastecer a todos (FAO, 2009). Nesse panorama, a entomofagia se mostra com um recurso estratégico. De acordo com a FAO, aproximadamente 2000 espécies de insetos são catalogadas como comestíveis (JONGEMA, 2017). No Brasil são 135 espécies registradas como apropriadas para alimentação, sobretudo espécies das ordens Hymenoptera, Coleoptera e Orthoptera (COSTA NETO e RAMOSELORDUY, 2006).

Dentre os insetos que podem ser utilizados na entomofagia estão as vespas sociais, conhecidas no Brasil como marimbondos ou cabas (Hymenoptera, Vespidae, Polistinae, Stenogastrinae e Vespinae (CARPENTER e MARQUES, 2001). No mundo há 974 espécies, sendo que o Brasil possui a maior diversidade, com $346 \mathrm{spp}$. (CARPENTER e ANDENA, 2013). Nessa perspectiva, o presente trabalho tem como objetivo uma revisão literária a fim de listar as espécies de vespas sociais de ocorrência no Brasil com potencial alimentício.

\section{MATERIAL E MÉTODOS}

O estudo foi realizado entre agosto de 2019 a junho de 2020 e consistiu na revisão literária de vespas sociais comestíveis de ocorrência no Brasil. Para o estudo, foram analisados artigos publicados, dissertações, teses e capítulos de livros relacionados à temática.

Os arquivos utilizados como referência no presente estudo foram obtidos das seguintes plataformas de pesquisa: Scielo, Scopus, Google Scholar e Research Gate, com registro de cerca de 30 artigos. Destes, foram utilizados 15, os quais foram os mais relacionados à entomofagia e às vespas, com destaque para o estudo de Jongema (2017).

\section{RESULTADOS E DISCUSSÃO}

No Brasil há ocorrência de seis espécies de vespas sociais que são utilizadas como fonte de alimento por povos indígenas (COSTA NETO e RAMOS-ELORDUY, 2006) (Quadro 1), bem como outras 34 espécies que são relatadas como comestíveis em outros países (JONGEMA, 2017) e que, portanto, têm potencial de uso no país (Quadro 2). No artigo de revisão realizado por Costa Neto e Ramos-Elorduy (2006), há o registro do uso de larvas e pupas de três vespas (espécies não identificadas) pelos índios Tapirapé, Desâna e Ianomâmi. Ressalta-se que além das espécies listadas, utilizadas como alimento, outros vespídeos de hábitos solitários podem possuir esse potencial devido aos seus aspectos similares de ecologia, fisiologia e morfologia. Ainda, ocorre o consumo de mel produzido por Brachygastra lecheguana (Latreille, 1824) como medicina popular (COSTA NETO et al., 2006). 
Quadro 1: Vespas sociais (Hymenoptera, Vespidae) utilizadas como fonte de alimento por diferentes povos indígenas do Brasil.

\begin{tabular}{|l|l|l|l|}
\hline Espécie & Estado consumido & Etnia & Localidade \\
\hline Apoica pallens (Fabricius, 1804) & Larva e pupa & Nhambiquara & Mato Grosso \\
\hline Brachygastra lecheguana (Latreille, 1824) & Larva, pupa e “mel” & Guarani M'byá & São Paulo \\
\hline Epipona quadrituberculata (Griboldo, 1892) & Larva e pupa & Nhambiquara & Mato Grosso \\
\hline Polybia dimidiata (Olivier, 1791) & Larva e pupa & Nhambiquara & Mato Grosso \\
\hline Polybia occidentalis (Olivier, 1791) & Pupa, larva e “mel” & Nhambiquara & Mato Grosso \\
\hline Pseudopolybia vespiceps (Saussure, 1864) & Larva e pupa & Nhambiquara & Mato Grosso \\
\hline
\end{tabular}

Fonte: Adaptado de Costa Neto et al. (2006).

Quadro 2: Espécies de vespas sociais com potencial de uso na alimentação encontradas no Brasil, Estado brasileiro de sua ocorrência e referências. As espécies já utilizadas como recurso entomofágico estão assinaladas com sinal de asterisco (*).

\begin{tabular}{|c|c|c|}
\hline Espécie & Estado de ocorrência & Referência \\
\hline $\begin{array}{l}\text { Agelaia angulata angulata } \\
\text { (Fabricius, 1804) }\end{array}$ & $\begin{array}{l}\text { AC, AM, AP, BA, ES, MA, MG, } \\
\text { MT, PA, SP, RO, RR, RJ, RS }\end{array}$ & $\begin{array}{l}\text { Richards (1978); Hermes e Kohler (2004); } \\
\text { Aragão e Andena (2016) }\end{array}$ \\
\hline Agelaia baeazae (Richards, 1943) & $\mathrm{AC}$ & Gomes et al. (2018) \\
\hline Agelaia lobipleura (Richards, 1978) & AM, MT, RO & Richards (1978); Gomes (2013) \\
\hline Agelaia ornata (Ducke, 1905) & $\mathrm{AM}, \mathrm{RO}$ & Richards (1978); Gomes (2013) \\
\hline Agelaia pallipes (Olivier, 1791) & $\begin{array}{l}\text { AC, BA, AM, CE, GO, MA, MG, } \\
\text { MT, MS, PA, PI, PR, RJ, RO, RN, } \\
\text { RR, RS, SP }\end{array}$ & $\begin{array}{l}\text { Richards (1978); Hermes e Kohler (2004); } \\
\text { Gomes (2013); Andena e Carpenter (2014); } \\
\text { Da Rocha e Silveira (2014); Pereira- } \\
\text { Bomfim, 2014; Virgínio et al. (2016); Souza } \\
\text { et al. (2017) }\end{array}$ \\
\hline $\begin{array}{l}\text { Angiopolybia paraensis } \\
\text { (Spinola, 1851) }\end{array}$ & AM, BA, PA, MA, MT, RO & $\begin{array}{l}\text { Richards (1978); Silva e Silveira (2009); } \\
\text { Gomes (2013); Andena e Carpenter (2014); } \\
\text { Somavilla et al. (2014) }\end{array}$ \\
\hline Apoica pallens (Fabricius, 1804)* & $\begin{array}{l}\text { AM, AP, BA, DF, GO, MA, MG, } \\
\text { MT, MS, PA, PI, PR, RJ, RO, RS, } \\
\text { SC, SP }\end{array}$ & $\begin{array}{l}\text { Richards (1978); Silveira et al. (2008); } \\
\text { Santos et al. (2009); Pereira e Antonialli-jr } \\
\text { (2011); Andena e Carpenter (2014); } \\
\text { Somavilla et al. (2014); Singling (2015); } \\
\text { Gomes et al. (2016); Raw (2016); Souza et } \\
\text { al. (2017) }\end{array}$ \\
\hline Apoica pallida (Olivier, 1791) & $\begin{array}{l}\text { AC, AM, AP, BA, CE, MA, MT, } \\
\text { PA, RO, RR, SP }\end{array}$ & Richards (1978); Neto e Andena (2011) \\
\hline Apoica strigata (Richards, 1978) & AC, AM, MA, PA & Richards (1978) \\
\hline Apoica thoracica (Buysson, 1906) & $\begin{array}{l}\text { AC, AM, AP, DF, ES, GO, MG, } \\
\text { MT, PA, PR, RO, RR, SP }\end{array}$ & $\begin{array}{l}\text { Richards (1978); Raw (1998); Gomes et al. } \\
\text { (2016); Raw (2016) }\end{array}$ \\
\hline $\begin{array}{l}\text { Brachygastra lecheguana (Latreille, } \\
\text { 1824)* }\end{array}$ & $\begin{array}{l}\text { AM, AP, BA, CE, DF, ES, GO, } \\
\text { MA, MG, MS, MT, PA, PB, PI, } \\
\text { PE, PR, RO, RJ, RN, RR, RS, SC, } \\
\text { SP }\end{array}$ & $\begin{array}{l}\text { Richards (1978); Hermes e Kohler (2004); } \\
\text { Da Rocha e Silveira (2014); Carbonari } \\
\text { (2009);Gomes (2013); Andena e Carpenter } \\
\text { (2014); Da Rocha e Silveira (2014) Raw } \\
\text { (2016); Elisei et al. (2017); Souza et al. } \\
\text { (2017) }\end{array}$ \\
\hline $\begin{array}{l}\text { Epipona quadrituberculata } \\
\text { (Griboldo, 1892)* }\end{array}$ & GO, MT, PA & Richards (1978) \\
\hline $\begin{array}{l}\text { Mischocyttarus rotundicollis } \\
\text { (Cameron, 1912) }\end{array}$ & $\begin{array}{l}\text { AM, AL, BA, ES, GO MG, MT, } \\
\text { PA, PR, RJ, RS, SC, SP }\end{array}$ & Richards (1978); Barbosa et al. (2018) \\
\hline $\begin{array}{l}\text { Mischocyttarus tomentosus } \\
\text { Zikán, } 1935\end{array}$ & PA, BA, RO & $\begin{array}{l}\text { Silveira (2002); Gomes (2013); Aragão e } \\
\text { Andena (2016) } \\
\end{array}$ \\
\hline Polistes bicolor (Lepeleitier, 1836) & $\mathrm{AC}, \mathrm{AM}, \mathrm{AP}, \mathrm{MG}, \mathrm{MT}, \mathrm{PA}$ & Richards (1978) \\
\hline Polistes canadensis (Linneu, 1758) & $\begin{array}{l}\text { AM, BA, GO, MA, MG, MT, MS, } \\
\text { PA, PI, PE, PB, RN, RR, SP }\end{array}$ & $\begin{array}{l}\text { Richards (1978); Torres et al. (2009); } \\
\text { Andena e Carpenter (2014); Da Rocha e } \\
\text { Silveira (2014); Virgínio et al. (2016); } \\
\text { Souza et al. (2017) } \\
\end{array}$ \\
\hline $\begin{array}{l}\text { Polistes carnifex carnifex } \\
\text { (Fabricius, 1775) }\end{array}$ & $\begin{array}{l}\text { AM, BA, ES, GO, MA, MG, MT, } \\
\text { MS, PA, PE, PI, PR, RJ, RN, RS, } \\
\text { SP }\end{array}$ & $\begin{array}{l}\text { Richards (1978); Virgínio et al. (2016); } \\
\text { Souza et al. (2017) }\end{array}$ \\
\hline
\end{tabular}




\begin{tabular}{|c|c|c|}
\hline Espécie & Estado de ocorrência & Referência \\
\hline Polistes cavapyta (Saussure, 1853) & RS & Hermes e Kohler (2006) \\
\hline Polistes deceptor (Schulz, 1905) & AC, AM, PA, RS & $\begin{array}{l}\text { Richards (1978); Somavilla et al. (2010); } \\
\text { Gomes et al. (2018) } \\
\end{array}$ \\
\hline $\begin{array}{l}\text { Polistes erythrocephalus } \\
\text { (Latreille, } 1813 \text { ) }\end{array}$ & $\begin{array}{l}\text { AC, AM, DF, GO, MG, MT, PA, } \\
\text { PR, RJ, SP }\end{array}$ & $\begin{array}{l}\begin{array}{l}\text { Richards (1978); Raw (2016); Souza et al. } \\
\text { (2017) }\end{array} \\
\end{array}$ \\
\hline Polistes major (Beauvois, 1818) & AM, ES, MT, PR, SP & Richards (1978) \\
\hline Polistes occipitalis (Ducke, 1904) & $\begin{array}{l}\text { AM, AP, DF, ES, MG, PA, RJ, } \\
\text { RO, SP }\end{array}$ & $\begin{array}{l}\text { Richards (1978); Gomes (2013); Raw } \\
\text { (2016); Souza et al. (2017) }\end{array}$ \\
\hline Polistes pacificus Fabricius, 1804 & $\begin{array}{l}\text { AC, AM, AP, BA, ES, MA, MG, } \\
\text { MT, PA, RS, SP }\end{array}$ & $\begin{array}{l}\text { Richards (1978); Diniz e Kitayama (1998); } \\
\text { Hermes e Kohler (2006); Souza e Prezoto } \\
\text { (2006); Gomes et al. (2018) }\end{array}$ \\
\hline Polistes testaceicolor Bequaert, 1937 & AC, AM, AP, MA, MT, PA, RO & Richards (1978); Somavilla et al. (2014) \\
\hline Polistes versicolor (Olivier, 1791) & $\begin{array}{l}\text { AC, AM, AP, BA, DF, ES, GO, } \\
\text { MA, MG, MS, MT, PA, PR, RJ, } \\
\text { RN, RO, RR, RS, SC, SP }\end{array}$ & $\begin{array}{l}\text { Richards (1978); Raw (1998); Santos et al. } \\
\text { (2009); Virgínio et al. (2016); Raw (2016); } \\
\text { Souza et al. (2017) }\end{array}$ \\
\hline Polybia diguetana (Buysson, 1905) & RO & Gomes (2013) \\
\hline Polybia dimidiata (Olivier, 1791)* & $\begin{array}{l}\text { AM, AC, AP, BA, DF, ES, GO, } \\
\text { MA, MG, MT, PA, RJ, RO, RR, } \\
\text { RS, SP }\end{array}$ & $\begin{array}{l}\text { Richards (1978); Raw (2016); Gomes et al. } \\
\text { (2018) }\end{array}$ \\
\hline Polybia emaciata (Saussure, 1854) & $\begin{array}{l}\mathrm{AC}, \mathrm{AM}, \mathrm{AP}, \mathrm{DF}, \mathrm{GO}, \mathrm{MG}, \mathrm{MT}, \\
\mathrm{PA}, \mathrm{RJ}, \mathrm{RO}, \mathrm{SP}\end{array}$ & Richards (1978); Raw (2016) \\
\hline Polybia flavifrons Smith, 1857 & $\begin{array}{l}\text { BA, DF, MT, MG, RJ, RN, RS, } \\
\text { SC, SP }\end{array}$ & $\begin{array}{l}\text { Richards (1978); Diniz e Kitayama (1998); } \\
\text { Mechi (2005); Somavilla et al. (2010); } \\
\text { Andena e Carpenter (2014); Raw (2016); } \\
\text { Souza et al. (2018) }\end{array}$ \\
\hline Polybia ignobilis (Haliday, 1836) & $\begin{array}{l}\text { AM, BA, CE, DF, ES, GO, MA, } \\
\text { MG, MT, MS, PA, PB, PE, PI, PR, } \\
\text { RJ, RN, RO, RR, RS,SC, SP }\end{array}$ & $\begin{array}{l}\text { Richards (1978); Raw (1998); Santos et al. } \\
\text { (2009); Somavilla et al. (2010); Da Rocha e } \\
\text { Silveira (2014); Pereira-Bomfim (2014); } \\
\text { Raw (2016); Souza et al. (2017) }\end{array}$ \\
\hline Polybia micans Ducke, 1904 & $\begin{array}{l}\text { AM, AP, BA, MA, MT, PA, RO, } \\
\text { RR }\end{array}$ & $\begin{array}{l}\text { Richards (1978); Gomes (2013); Andena e } \\
\text { Carpenter (2014) }\end{array}$ \\
\hline $\begin{array}{l}\text { Polybia occidentalis (Olivier, } \\
\text { 1791)* }\end{array}$ & $\begin{array}{l}\text { AM, BA, CE, DF, ES, GO, MA, } \\
\text { MG, MT, MS, PA, PB, PE, PI, PR, } \\
\text { RJ, RN, RO, RR, RS, SC, SP }\end{array}$ & $\begin{array}{l}\text { Richards (1978); Lorenzato (1985); Souza e } \\
\text { Zanuncio (2012); Da Rocha e Silveira } \\
\text { (2014); Pereira-Bomfim (2014); Raw (2016) }\end{array}$ \\
\hline Polybia parvulina Richards, 1970 & $\mathrm{AM}, \mathrm{AP}, \mathrm{PA}, \mathrm{RO}, \mathrm{RR}$ & $\begin{array}{l}\text { Richards (1978); Silveira (2012); Gomes } \\
\text { (2013) }\end{array}$ \\
\hline Polybia rejecta (Fabricius, 1798) & $\begin{array}{l}\text { AC, AM, AP, BA, DF, ES, GO, } \\
\text { MA, MG, MT, PA, PE, RJ, RN, } \\
\text { RO, RR, SP }\end{array}$ & $\begin{array}{l}\text { Richards (1978); Raw (1998); Souza et al. } \\
\text { (2012); Virgínio et al. (2016); Raw (2016) }\end{array}$ \\
\hline Polybia ruficeps (Schrottky, 1902) & $\begin{array}{l}\text { BA, CE, DF, GO, MA, MG, MT, } \\
\text { MS, PI, RN, SC, SP }\end{array}$ & $\begin{array}{l}\text { Richards (1978); Tanaka e Noll (2011); } \\
\text { Andena e Carpenter (2014); Da Rocha e } \\
\text { Silveira (2014); Pereira-Bomfim (2014); } \\
\text { Souza et al. (2015); Raw (2016); Virgínio et } \\
\text { al. (2016) }\end{array}$ \\
\hline Polybia sericea (Olivier, 1791) & $\begin{array}{l}\text { AM, AP, BA, CE, DF, GO, MA, } \\
\text { MG, MT, MS, PA, PB, PI, PR, RJ, } \\
\text { RN, RO, RS, SC, SP }\end{array}$ & $\begin{array}{l}\text { Richards (1978); Diniz e Kitayama (1998); } \\
\text { Gomes (2013); Da Rocha e Silveira (2014); } \\
\text { Pereira-Bomfim (2014); Raw (2016) }\end{array}$ \\
\hline Polybia striata (Fabricius, 1787) & $\begin{array}{l}\text { AC, AM, AP, ES, GO, MA, MG, } \\
\text { MT, PA, RJ, RR, RO, SP }\end{array}$ & $\begin{array}{l}\text { Richards (1978); Souza et al. (2012); Gomes } \\
\text { (2013) }\end{array}$ \\
\hline $\begin{array}{l}\text { Pseudopolybia vespiceps vespiceps } \\
\text { (Saussure, } 1864) *\end{array}$ & $\begin{array}{l}\text { AC, BA, DF, ES, GO, MA, MG, } \\
\text { MT, PA, PE, PR, RJ, RO, RR, SP }\end{array}$ & $\begin{array}{l}\text { Richards (1978); Diniz e Kitayama (1998); } \\
\text { Silva e Silveira (2009); Souza et al. (2012); } \\
\text { Gomes (2013); Somavilla et al. (2014); } \\
\text { Andena e Carpenter (2014); Raw (2016); } \\
\text { Gomes et al. (2018) }\end{array}$ \\
\hline Synoeca surinama (L. 1767) & $\begin{array}{l}\text { AM, AP, BA, DF, GO, MA, MG, } \\
\text { MS, MT, PA, PB, PE, RJ, RO, RR, } \\
\text { SP }\end{array}$ & $\begin{array}{l}\text { Richards (1978); Raw (1998); Silveira et al. } \\
\text { (2008); Tanaka e Noll (2011); Grandinete e } \\
\text { Noll, (2013); Gomes (2013); Raw (2016); } \\
\text { Souza et al. (2017) }\end{array}$ \\
\hline Synoeca virginea (Fabricius, 1804) & $\begin{array}{l}\text { AC, AM, AP, MA, MT, PA, PI, } \\
\text { PE, RO, RR }\end{array}$ & $\begin{array}{l}\text { Richards (1978); Raw (1998); Silveira et al. } \\
\text { (2008); Gomes (2013); Andena e Carpenter } \\
\text { (2014) }\end{array}$ \\
\hline
\end{tabular}


Brachygastra lecheguana é utilizada por algumas etnias indígenas brasileiras, que consomem o "mel" produzido por essa espécie, além das larvas e pupas (COSTA NETO e RAMOS-ELORDUY, 2006). Esse hábito é registrado para os seguintes grupos: Guarani M’byá, residentes no Estado de São Paulo; Tapirapé, habitantes do nordeste do Mato Grosso; Desâna, residentes do noroeste do Estado do Amazonas; Ianomâmi, indígenas caçadores-agricultores com grupos na Venezuela e no Brasil, ocupando áreas dos Estados de Roraima e Amazonas; e Enawenê-Nawê, que possuem uma única aldeia no noroeste do Mato Grosso (COSTA NETO et al., 2006). No entanto, essa caba possui ampla distribuição no Brasil, sendo registrada em 23 Estados (RICHARDS, 1978; HERMES e KOHLER, 2004; DA ROCHA e SILVEIRA, 2014; CARBONARI, 2009; GOMES, 2013; ANDENA e CARPENTER, 2014; DA ROCHA e SILVEIRA, 2014; RAW, 2016; ELISEI et al., 2017; SOUZA et al., 2017). Essa espécie também possui ocorrência no México, onde seu mel é comercializado pelos povos indígenas Tzeltzales e Tzoltziles, que também consomem seus ovos, larvas e pupas (RAMOSELORDUY et al., 2002).

Polybia occidentalis é consumida pelas etnias Tzeltales, Tzoltziles e Tojolabales no sudoeste do México (RAMOS-ELORDUY e PINO MORENO, 2002). Estes grupos indígenas, localizados no chamado Alto de Chiapas, conservam práticas ancestrais Maias e fazem uso de diversos insetos na alimentação. No Brasil. os índios Guarani M'byá consomem o "mel” estocado por essa espécie em seu ninho (COSTA NETO e RAMOS-ELORDUY, 2006). Já os Tapirapé do rio Uaupés, os Ianomâmi, os Enawenê-Nawê e os Nhambiquara consomem larvas e pupas (COSTA NETO e RAMOS-ELORDUY, 2006). Sua ocorrência no território brasileiro é ampla, sendo registrada em 23 Estados (RICHARDS, 1978; LORENZATO, 1985; SOUZA e ZANUNCIO, 2012; DA ROCHA e SILVEIRA, 2014; PEREIRA-BOMFIM, 2014; RAW, 2016).

Pseudopolybia vespiceps, Polybia dimidiata e Epipona quadrituberculata são espécies consumidas na forma de larva ou pupa pelos índios Nhambiquara, tribo que possui aldeias nos Estados de Mato Grosso e Rondônia, que também fazem uso de outros insetos em sua dieta, como formigas, abelhas, besouros, gafanhotos, borboletas, mariposas e mosquitos (COSTA NETO e RAMOS-ELORDUY, 2006). Esses índios habitam tanto o Cerrado quanto a floresta Amazônica, além do ecótono entre esses dois ecossistemas. Os Nhambiquara ficaram conhecidos pelos estudos do antropólogo Claude LéviStrauss, por terem sido contatados pelo Marechal Cândido Rondon (MILLER, 2008). Essas três espécies de cabas estão amplamente distribuídas no território brasileiro.

Apoica pallens é uma vespa social de hábito noturno, utilizada na alimentação humana no estágio de pupa e larva por diversas etnias indígenas. No Mato Grosso, os Nhambiquara e os Tapirapé possuem o hábito de consumir essa espécie. No Amazonas, os Desâna e os Yanomani também utilizam esse inseto na dieta (COSTA NETO e RAMOS-ELORDUY, 2006). Essa vespa é cosmopolita no Brasil, ocorrendo em 17 Estados (RICHARDS, 1978; SILVEIRA et al., 2008; SANTOS et al., 2009; PEREIRA e ANTONIALLI-JR, 2011; ANDENA e CARPENTER, 2014; SOMAVILLA et al., 2014; SINGLING, 2015; GOMES et al., 2016; RAW, 2016; SOUZA et al., 2017). Há registro do uso alimentar dessa espécie em algumas regiões do Equador (ONORE, 2005).

Muitas pessoas, principalmente de países ocidentais, consideram o consumo de insetos como prática ultrapassada, alimentação de pessoas pobres e marginalizadas. Além disso, algumas culturas consideram a maioria dos insetos como animais nocivos, sujos, transmissores de doenças e vistos como pragas (GARINE, 1987; KELLERT, 1993; VANHONACKER et al., 2013), o que afeta negativamente a prática da entomofagia. Entretanto, os benefícios ecológicos e nutricionais do uso dos insetos na alimentação são promissores, por isso a entomofagia deve ser considerada como alternativa econômica e socioambiental.

\section{CONCLUSÕES}

A região amazônica abriga o maior número de espécies de vespídeos do país. O bioma possui ainda o maior número de espécies de vespas sociais comestíveis, o que torna a área de alto potencial para a entomofagia por populações não indígenas.

O uso sustentável de insetos comestíveis pode beneficiar a conservação dos recursos naturais, e, assim, desempenhar um papel importante, seja na melhoria da alimentação humana ou na conservação da biodiversidade. Entretanto, esse patrimônio natural está ameaçado pelo contínuo desmatamento, poluição, exploração exacerbada do solo e outros impactos antrópicos, sendo necessária a implantação de modelos de produção ou de desenvolvimento econômico que sejam de fato sustentáveis. 


\section{REFERÊNCIAS}

Andena, S. R.; Carpenter, J. M. Checklist das espécies de Polistinae (Hymenoptera, Vespidae) do semiárido brasileiro. In: Bravo, F.; Calor, A. (eds.). Artrópodes do semiárido, biodiversidade e conservação. Feira de Santana: Printmídia, 2014. p.169-180.

Aragão, M.; Andena, S. R. The social wasps (Hymenoptera: Vespidae: Polistinae) of a fragment of Atlantic Forest in southern Bahia, Brazil. Journal of Natural History, v. 50, p. 1411-1426, 2016.

Banjo, A. D. et al. Ethno-zoological knowledge and perception of the value of insects among the Ijebus (south western Nigeria). Global Journal of Pure and Applied Sciences, v. 10, n. 1, p. 1-6, 2004.

Barbosa, B. C.; Marciel, T. T.; Prezoto, F. New records of social wasps (Hymenoptera: Vespidae: Polistinae) in Alagoas state, Brazil. EntomoBrasilis, v. 11, n. 1, p. 56-59, 2018.

Carbonari, V. Composição faunística de vespas (Hymenoptera: Apocrita) do Parque Nacional da Serra da Bodoquena. 2009. 54 f. Dissertação de Mestrado, Universidade Federal da Grande Dourados, Dourados, Mato Grosso do Sul, 2009.

Carpenter, J. M.; Marques O. M. Contribuição ao estudo dos vespídeos do Brasil. Salvador: Série Publicações Digitais, 2001

Carpenter J. M.; Andena S. R. The Vespidae of Brazil. Manaus: Instituto Nacional de Pesquisa da Amazônia, 2013.

Costa Neto, E. M. Insetos no cardápio. Ciência Hoje, v. 27, p. 63-65, 2000.

Costa Neto, E. M. Manual de etnoentomología. Zaragoza: Manuales \& Tesis SEA, 2002.

Costa Neto E. M. Insetos como fontes de alimentos para o homem: valoração de recursos considerados repugnantes. Interciencia, v. 28, n. 3, p. 136-140, 2003.

Costa Neto, E. M. Estudos etnoentomológicos no estado da Bahia, Brasil: uma homenagem aos 50 anos do campo de pesquisa. Biotemas, v. 17, n. 1, p. 117-149, 2004.

Costa Neto, E. M.; Ramos-Elorduy, J. Los insectos comestibles de Brasil: etnicidad, diversidad e importancia en la alimentación Boletín de la Sociedad Entomológica Aragonesa, v. 38, p. 423-442, 2006.

Costa Neto, E. M.; Ramos-Elorduy, J.; Pino, J. M. M. Los insectos medicinales de Brasil: primeros resultados. Boletín de la Sociedad Entomológica Aragonesa, v. 38, p. 395-414, 2006.

Da Rocha, A. A.; Silveira, O. T. Current knowledge about the social wasps (Hymenoptera: Vespidae) in the state of Piauí, Brazil. EntomoBrasilis, v. 7, n. 2, p. 167-170, 2014.

Diniz, I. R.; Kitayama, K. Seasonality of vespid species (Hymenoptera: Vespidae) in a central Brazilian cerrado. Biol. Trop., v. 46, p. 109-114, 1998.

Elisei, T.; Valadares, E.; Albuquerque, F. A.; Martins, C. F. Diversity and structure of social wasps community (Hymenoptera: Vespidae, Polistinae) in neotropical dry forest. Embrapa Algodão-Artigo em periódico indexado (ALICE). Sociobiology, v. 64, n. 1, p. 111-118, 2017.

FAO, Food and Agriculture Organization of the United Nations. Global agriculture towards 2050. Rome: FAO, 2009.

Garine, I. Alimentação, culturas e sociedades. O Correio da Unesco, v. 15, n. 7, p. 4-7, 1987.

Gomes, B. Diversidade de vespas sociais (Vespidae, Polistinae) na região norte de Rondônia e relação dos ciclos ambientais abióticos sobre o forrageio. 2013. 55f. Tese, Universidade de São Paulo, Ribeirão Preto, São Paulo, 2013.

Gomes, B.; da Silva, C. L. A.; da Silva, M.; Noll, F. B. Survey and new distributional records of nocturnal social wasps Apoica (Hymenoptera, Vespidae, Epiponini) along Madeira River, Rondônia, Brazil. EntomoBrasilis, v. 9, n. 1, p. 59-61, 2016.

Gomes, B.; Knidel, S. V. D. L.; Moraes, H. D. S.; Silva, M. D. Survey of social wasps (Hymenoptera, Vespidae, Polistinae) in Amazon rainforest fragments in Acre, Brazil. Acta Amazonica, v. 48, n. 2), p. 109-116, 2018.

Grandinete, Y. C.; Noll, F. B. Checklist of social (Polistinae) and solitary (Eumeninae) wasps from a fragment of cerrado "campo sujo" in the State of Mato Grosso do Sul. Sociobiology, v. 60, p. 101-106, 2013.

Hermes M. G.; Köhler, A. The flower-visiting social wasps (Hymenoptera, Vespidae, Polistinae) in two areas of Rio Grande do Sul State, Southern Brazil. Revista Brasileira de Entomologia, v. 50, p. 268-274, 2006.

Hermes, M. G.; Köhler, A. Chave ilustrada para as espécies de Vespidae (Insecta, Hymenoptera) ocorrentes no Cinturão Verde de Santa Cruz do Sul, RS, Brasil. Caderno de Pesquisa-Série Biologia, v. 16, n. 2, p. 65$115,2004$.

Jongema, Y. List of edible insects of the world (April 1, 2017). Laboratory of Entomology, Wageningen University, Wageningen. Disponível em: https://www.wur.nl/en/Research-Results/Chair-groups/PlantSciences/Laboratory-of-Entomology/Edible-insects/Worldwide-species-list.htm.

Kellert, S. R. Values and perceptions of invertebrates. Conservation Biology, v. 7, n. 4, p. 845-853, 1993.

Lorenzato, D. Ocorrência e flutuação populacional de abelhas e vespas em pomares de macieiras Malus domestica Bork e pessegueiros Prunus persica Zucc. no alto vale do Rio do Peixe, Santa Catarina e eficiência de atrativos alimentares sobre esses hymenopteros. Agronomia Sulriograndense, v. 21, p. 87-109, 1985. 
Malaisse, F. Se nourrir en forêt claire. Approche écologique et nutritionnelle. Wageningen: Presses Agronomiques de Gembloux, 1997.

Mechi, M. R. Comunidade de vespas Aculeata (Hymenoptera) e suas fontes florais. In: Pivello, V. R.; Varanda, E. M. (eds.). O cerrado Pé-de-Gigante: ecologia e conservação - Parque Estadual de Vassununga. São Paulo: Secretaria do Meio Ambiente, 2005. p. 256-266.

Nonaka, K. Ethnoentomology of the Central Kalahari San. Afr Study Monogr (Supplementary Issue), v. 22, p. 29-46, 1996

Miller, J. As coisas: os enfeites corporais e a noção de pessoa entre os Mamaindê (Nambiquara). Rio de Janeiro: Mauad Editora Ltda, 2008.

Neto, A. M. S.; Andena, S. R. New records of Apoica pallida (Olivier, 1792) (Hymenoptera: Vespidae, Epiponini) in Bahia State. Entomobrasilis, v. 4, n. 3, p. 152-153, 2011.

Onore, G. Edible insects in Ecuador. In: Paoletti, M. et al. (ed). Ecological implications of minilivestock: Insects, rodents, frogs and snails. New Hampshire: Science Publiscers Inc, 2005. p. 343-352.

Pereira, M. D. G. C.; Antonialli-Junior, W. F. Social wasps in riparian forest in Batayporã, Mato Grosso do Sul state, Brazil. Sociobiology, v. 57, p. 153-163, 2011.

Pereira-Bomfim, M. G. C. Community structure of social wasps (Hymenoptera: Vespidae) in riparian forest in Batayporã, Mato Grosso do Sul, Brazil. Sociobiology, v. 59, n. 3, p. 755-765, 2014.

Ramos-Elorduy, J.; Pino Moreno, J. M. Edible insects of Chiapas, Mexico. Ecology of Food and Nutrition, v. 41, n. 4, p. 271-299, 2002.

Ramos-Elorduy, J. La etnoentomología en la alimentación, la medicina y el reciclaje. In: Biodiversidad, taxonomía y biogeografía de artrópodos de México: hacia una síntesis de su conocimiento. México: UNAM, 2004. p. 329-413.

Ramos-Elorduy, J.; Blasquez, J.; Pino Moreno, J. M.; Martinez Camacho, V.H. Could grasshoppers be a nutritive meal? Food and Nutrition Sciences, v. 3, p. 164-175, 2012.

Raw, A. Social wasps (Hymenoptera, Vespidae) of Ilha de Maracá. In: Ratte, J. A.; Milliken, W. (eds.). The biodiversity and environment of an Amazonian rainforest. Edimburgo: Royal Botanic Garden Edinburgh, 1998. p 307-321.

Raw, A. New records of social wasps around Brasília (Hymenoptera; Vespidae; Polistinae). Sociobiology, v. 63, n. 4, p. 1073-1075, 2016.

Richards, O.W. The social wasps of the Americas. Londres: Brit. Museum (Nat. Hist), 1978.

Ricklefs, R. E. A. Economia da natureza: um livro-texto em ecologia básica. Rio de Janeiro: Guanabara Koogan, 1996.

Romero, L. Alimentação. Por que você deve começar a comer insetos. Revista Super Interessante, n. 312, p. 1-4, 2012.

Santos, G. M. M.; Cruz, J. D. da; Marques, O. M.; Gobbi, N. Diversidade de vespas sociais (Hymenoptera: Vespidae) em áreas de Cerrado na Bahia. Neotropical Entomology, n. 38, p. 317-320, 2009.

Silva, S. de S.; Silveira, O. T. Vespas sociais (Hymenoptera, Vespidae, Polistinae) de floresta pluvial Amazônica de terra firme em Caxiuanã, Melgaço, Pará. Iheringia, Série Zoologia, v. 99, n. 3, p. 317-323, 2009.

Silveira, O. T.; Costa Neto, S. V. D.; Silveira, O. F. M. D. Social wasps of two wetland ecosystems in Brazilian Amazonia (Hymenoptera, Vespidae, Polistinae). Acta Amazonica, v. 38, p. 333-344, 2008.

Silveira, O. T. Surveying Neotropical social wasps. An evaluation of methods in the "Ferreira Penna" Research Station (ECFPn), in Caxiuana, PA, Brazil (Hymenoptera, Vespidae, Polistinae). Papéis Avulsos de Zoologia, v. 42, p. 299-323, 2002.

Singling, H. L. V. Inventário de vespas sociais (Hymenoptera, Vespidae) e comparação da eficiência de armadilhas atrativas em diferentes estratos vegetais na Floresta Estadual Edmundo Navarro de Andrade. 2015. 31f. Trabalho de conclusão de curso de graduação. Rio Claro, São Paulo, 2015.

Sirimungkararat, S.; Saksirirat, W.; Nopparat, T.; Natongkham, A. Edible products from eri and mulberry silkworms in Thailand. In: Patrick, B. D. (ed.). Forest insects as food: humans bite back. Chiang Mai: Food and Agriculture Organization of the United Nations, Regional Office for Asia and the Pacific, 2010. p. 189-200

Somavilla, A.; Köhler, A.; Hermes, M. G. Contribuição aos estudos dos Vespidae ocorrentes no estado do Rio Grande do Sul (Insecta, Hymenoptera). Rev. Bras. Bioci., v. 8, n. 3, p. 257-263, 2010.

Somavilla, A.; Oliveira, M. L.; Silveira, O. T. Diversity and aspects of the ecology of social wasps (Vespidae, Polistinae) in Central Amazonian" terra firme" forest. Revista Brasileira de Entomologia, v. 58, n. 4, p. 349-355, 2014

Souza, M. M.; Prezoto, F. Diversity of social wasps (Hymenoptera, Vespidae) in Semideciduous forest and cerrado (savanna) regions in Brazil. Sociobiology, v. 47, n. 1, p. 135-147, 2006.

Souza, M. M.; Zanuncio, J. C. Marimbondos: vespas sociais (Hymenoptera: Vespidae). Viçosa: Editora UFV, 2012.

Souza, M. M. et al. Biodiversidade de vespas sociais (Hymenoptera: Vespidae) do Parque Estadual do Rio Doce, Minas Gerais, Brasil. MG. Biota, v. 5, p. 04-19, 2012. 
Souza, M. M. et al. Biodiversity of social wasps (Hymenoptera: Vespidae) at altitudes above 1600 meters in the Parque Estadual da Serra do Papagaio, State of Minas Gerais, Brazil. EntomoBrasilis, v. 8, n. 3, p. 174-179, 2015.

Souza, M. M.; Brunismann, A. G.; Clemente, M. A. Species composition, relative abundance and distribution of social wasps fauna on different ecosystems. Sociobiology, p. 456-465, 2017.

Souza, M. M.; Clemente, M. A.; Milani, L. R. Inventário de vespas sociais (Hymenoptera, vespidae) dos Parques Estaduais Serra do Papagaio e do Ibitipoca, Sudeste do Brasil. MG. Biota, v. 11, p. 32-42, 2018.

Tanaka. J. G. M.; Noll, F. B. Diversity of social wasps on semideciduous seasonal forest fragments with different surrounding matrix in Brazil. Psyche: A Journal of Entomology, v. 8, 2011.

Torres, V. D. O.; Montagna, T. D. S.; Bortoluzz, G.; Antonialli-Junior, W. F. Aspectos bionômicos da vespa social Neotropical Polistes canadensis canadensis (Linnaeus)(Hymenoptera, Vespidae. Revista Brasileira de Entomologia, v. 53, n. 1, p. 134-138, 2009.

Vanhonacker, F.; Van Loo, E. J.; Gellynck, X.; Verbeke, W. Flemish consumer attitudes towards more sustainable food choices. Appetite, v. 62, p. 7-16, 2013.

Van Huis, A. Cultural aspects of ants, bees and wasps and their products in sub-Saharan Africa. Research Square, 2020. DOI: 10.21203/rs.3.rs-33219/v1.

Van Huis, A. Potential of insects as food and feed in assuring food security. Annual Review of Entomology, v. 58, p. 563-583, 2013.

Virgínio, F.; Maciel, T. T.; Barbosa, B. C. Novas contribuições para o conhecimento de vespas sociais (Hymenoptera: Vespidae) para Estado do Rio Grande do Norte, Brasil. Entomotropica, v. 31, p. 221-226, 2016. 